Korean J. Math. 20 (2012), No. 4, pp. 485-491

http://dx.doi.org/10.11568/kjm.2012.20.4.485

\title{
ON THE NORM ATTAINING OPERATORS
}

\author{
JUN IK LEE
}

\begin{abstract}
In this paper, we show the norm attaining paranormal operators have a nontrivial invariant subspace. Also, we show the norm attaining quadratically hyponormal weighted shift is subnormal.
\end{abstract}

\section{Introduction}

Let $\mathcal{H}$ be a complex Hilbert space and let $\mathcal{B}(\mathcal{H})$ denote the algebra of bounded linear operators on $\mathcal{H}$. An operator $T \in \mathcal{B}(\mathcal{H})$ is said to be normal if $T^{*} T=T T^{*}$, hyponormal if $T^{*} T \geq T T^{*}$ and subnormal if $T=\left.N\right|_{\mathcal{H}}$, where $N$ is normal on some Hilbert space $\mathcal{K} \supseteq \mathcal{H}$. An operator $T \in \mathcal{B}(\mathcal{H})$ is said to be paranormal if $\left\|T^{2} x\right\| \geq\|T x\|^{2}$ for all unit vector $x \in \mathcal{H}$. An operator $T \in \mathcal{B}(\mathcal{H})$ is called norm attaining if there is an $x \in \mathcal{H}$ with $\|x\|=1$ and $\|T x\|=\|T\|$. The Bram-Halmos criterion for subnormality states that an operator $T$ is subnormal if and only if

$$
\sum_{i, j}\left(T^{i} x_{j}, T^{j} x_{i}\right) \geq 0
$$

Received November 5, 2012. Revised December 12, 2012. Accepted December 15, 2012.

2010 Mathematics Subject Classification: 47B20, 47A15, 47B37, 47A13.

Key words and phrases: invariant subspace problem, paranormal operators, norm attaining operators.

This research was supported by a 2011 Research Grant from Sangmyung University.

(c) The Kangwon-Kyungki Mathematical Society, 2012.

This is an Open Access article distributed under the terms of the Creative commons Attribution Non-Conercial License (http://creativecommons.org/licenses/bync/3.0/) which permits unrestricted non-commercial use, distribution and reproduction in any medium, provided the original work is properly cited. 
for all finite collections $x_{0}, x_{1}, \cdots, x_{k} \in \mathcal{H}([3],[6$, II.1.9]). It is easy to see that this is equivalent to the following positivity test:

$$
\left(\begin{array}{cccc}
I & T^{*} & \cdots & T^{* k} \\
T & T^{*} T & \cdots & T^{* k} T \\
\vdots & \vdots & \ddots & \vdots \\
T^{k} & T^{*} T^{k} & \cdots & T^{* k} T^{k}
\end{array}\right) \geq 0 \quad(\text { all } k \geq 1)
$$

Condition (0.1) provides a measure of the gap between hyponormality and subnormality. In fact, the positivity condition (0.1) for $k=1$ is equivalent to the hyponormality of $T$, while subnormality requires the validity of $(0.1)$ for all $k$. Let $[A, B]:=A B-B A$ denote the commutator of two operators $A$ and $B$, and define $T$ to be $k$-hyponormal whenever the $k \times k$ operator matrix

$$
M_{k}(T):=\left(\left[T^{* j}, T^{i}\right]\right)_{i, j=1}^{k}
$$

is positive. An application of the Choleski algorithm for operator matrices shows that the positivity of $(0.2)$ is equivalent to the positivity of the $(k+1) \times(k+1)$ operator matrix in $(0.1)$; the Bram-Halmos criterion can be then rephrased as saying that $T$ is subnormal if and only if $T$ is $k$-hyponormal for every $k \geq 1([9])$.

Recall $([1],[9],[7])$ that $T \in \mathcal{L}(\mathcal{H})$ is said to be weakly $k$-hyponormal if

$$
L S\left(T, T^{2}, \cdots, T^{k}\right):=\left\{\sum_{j=1}^{k} \alpha_{j} T^{j}: \alpha=\left(\alpha_{1}, \cdots, \alpha_{k}\right) \in \mathbb{C}^{k}\right\}
$$

consists entirely of hyponormal operators, or equivalently, $M_{k}(T)$ is weakly positive, i.e., ([9])

$$
\left\langle M_{k}(T)\left(\begin{array}{c}
\lambda_{1} x \\
\vdots \\
\lambda_{k} x
\end{array}\right),\left(\begin{array}{c}
\lambda_{1} x \\
\vdots \\
\lambda_{k} x
\end{array}\right)\right\rangle \geq 0 \text { for all } x \in \mathcal{H} \text { and } \lambda_{1}, \cdots, \lambda_{k} \in \mathbb{C} \text {. }
$$

If $k=2$ then $T$ is said to be quadratically hyponormal and if $k=3$ then $T$ is said to be cubically hyponormal. Similarly, $T \in \mathcal{L}(\mathcal{H})$ is said to be polynomially hyponormal if $p(T)$ is hyponormal for every polynomial $p \in \mathbb{C}[z]$. It is known that $k$-hyponormal $\Rightarrow$ weakly $k$-hyponormal, but the converse is not true in general.

The invariant subspace problem is the simple question: Does every bounded linear operator $T$ on a separable Hilbert space $\mathcal{H}$ over $\mathbb{C}$ have a 
non-trivial invariant subspace? Here non-trivial subspace means a closed subspace of $\mathcal{H}$ different from 0 and different from $\mathcal{H}$. Invariant means that the operator $T$ maps it to itself. The problem is easy to state, however, it is still open. The answer is no in general for (separable) complex Banach spaces. For certain classes of bounded linear operators on complex Hilbert spaces, the problem has an affirmative answer(for example, compact operators, isometry operators, subnormal operators etc). But, the problem is still open for operators on a separable complex Hilbert space.

On the other hand, the celebrated Bishop-Phelps theorem states that the set of norm attaining functionals on a Banach space is norm dense in the dual space([2]). J. Lindenstrauss ([15]) obtained for a reflexive space $X$ and any Banach space $Y$ that the norm attaining operators in $\mathcal{L}(X, Y)$ are dense and provided also the first counterexample: the set of norm attaining operators from $c_{0}$ into any strictly convex space containing $c_{0}$ is not dense in the set of all bounded operators. Nevertheless, there are also remarkable situations in which a Bishop-Phelps theorem for operators does hold, such as when the domain space is reflexive ([15]) or, more generally, when it has the Radon-Nikodym property ([4]). After the pioneering paper by Lindenstrauss, several results dealing with denseness of norm attaining operators appeared ([4],[12],[13], etc). Since every Hilbert space is reflexive, norm attaining operators on a Hilbert space $\mathcal{H}$ are dense. In this paper, we will show the norm attaining paranormal operators have a nontrivial invariant subspace.

\section{Main results}

We start from a basic criterion for norm attaining operators.

LEMma 1. If $T \in \mathcal{B}(\mathcal{H})$ is a norm attaining operator if and only if $\|T\|^{2} \in \sigma_{p}\left(T^{*} T\right)$, where $\sigma_{p}(S)$ denote the point spectrum of $S \in \mathcal{B}(\mathcal{H})$.

Proof. Observe that $\|T x\|=\|T\|\|x\|$ if and only if $\left\langle\left(T^{*} T-\|T\|^{2}\right) x, x\right\rangle$ $=0$. Since $T^{*} T-\|T\|^{2}$ is hermitian, we can see that $\left\langle\left(T^{*} T-\|T\|^{2}\right) x, x\right\rangle=$ 0 if and only if $T^{*} T x=\|T\|^{2} x$ or equivalently, $x \in \operatorname{Ker}\left(T^{*} T-\|T\|^{2} I\right)$. Thus $T$ is a norm attaining operator if and only if $\|T\|^{2} \in \sigma_{p}\left(T^{*} T\right)$.

Remark 2. Note that if $T \in \mathcal{B}(\mathcal{H})$, then $\|T\|^{2} \in \sigma_{a p}\left(T^{*} T\right)$, where $\sigma_{a p}(S)$ denote the approximate point spectrum of $S \in \mathcal{B}(\mathcal{H})$. 
Proof. Since $T^{*} T$ is positive for any $T \in \mathcal{B}(\mathcal{H}), T^{*} T$ is normaloid(i.e., norm equals to spectral radius). Hence $\|T\|^{2}=\left\|T^{*} T\right\|$ is a spectral value of $T^{*} T$. From the fact that the boundary of the spectrum is contained in the approximate point spectrum([11]), the desired result follows.

We now have our main theorem:

Theorem 3. If $T \in \mathcal{B}(\mathcal{H})$ is a norm attaining paranormal operator then $T$ has a non-trivial invariant subspace, and hence norm attaining hyponormal operators have a non-trivial invariant subspace.

Proof. Let $M:=\{x \in \mathcal{H}:\|T x\|=\|T\|\|x\|\}$. Observe that $x \in M$ if and only if $x \in \operatorname{Ker}\left(\|T\|^{2} I-T^{*} T\right)$. Thus $M$ is a closed subspace of $\mathcal{H}$ and $M \neq\{0\}$ by the hypothesis. If $x \in M$ with $\|x\|=1$, then by the paranormality of $T$ we have

$$
\left\|T^{2} x\right\| \geq\|T x\|^{2}=(\|T\|\|x\|)^{2}=\|T\|^{2} .
$$

Thus

$$
\left\|T^{2} x\right\| \leq\left\|T \left|\||| T x\|=\|T\|^{2}\|x\|=\|T\|^{2} \leq\left\|T^{2} x\right\| .\right.\right.
$$

This shows that $\left\|T^{2} x\right\|=\|T\|\|T x\|$ for $x \in M$ with $\|x\|=1$ and hence $M$ is invariant under $T$. If $M \neq \mathcal{H}$, then $M$ is a non-trivial invariant subspace for $T$. If $M=\mathcal{H}$, then $\frac{T}{\|T\|}$ is an isometry and hence $T$ has a non-trivial invariant subspace.

Recall that given a bounded sequence of positive real numbers $\alpha$ : $\alpha_{0}, \alpha_{1}, \cdots$ (called weights), the (unilateral) weighted shift $W_{\alpha}$ associated with $\alpha$ is the operator on $\ell^{2}\left(\mathbb{Z}_{+}\right)$defined by $W_{\alpha} e_{n}:=\alpha_{n} e_{n+1}$ for all $n \geq 0$, where $\left\{e_{n}\right\}_{n=0}^{\infty}$ is the canonical orthonormal basis for $\ell^{2}\left(\mathbb{Z}_{+}\right)$ (where $\mathbb{Z}_{+}$is the set of non-negative integers). It is straightforward to check that $W_{\alpha}$ can never be normal, and that $W_{\alpha}$ is hyponormal if and only if $\alpha_{n} \leq \alpha_{n+1}$ for all $n \geq 0$. We then have:

THEOREM 4. $W_{\alpha}$ is norm attaining if and only if $\left\|W_{\alpha}\right\|=\alpha_{i}$ for some $i$

Proof. Since $W_{\alpha}^{*} W_{\alpha}=\operatorname{diag}\left\{\alpha_{0}^{2}, \alpha_{1}^{2}, \cdots\right\}$, we have $\sigma_{p}\left(W_{\alpha}^{*} W_{\alpha}\right)=\left\{\alpha_{0}^{2}, \alpha_{1}^{2}\right.$, $\cdots\}$. The desired result now follows from Lemma 1 .

J. Stampfli [16] showed that for subnormal weighted shifts $W_{\alpha}$, a propagation phenomenon occurs which forces the flatness of $W_{\alpha}$ whenever two equal weights are present. Later, A. Joshi proved in [14] that the shift 
with weights $\alpha_{0}=\alpha_{1}=a, \alpha_{2}=\alpha_{3}=\cdots=b, 0<a<b$, is not quadratically hyponormal, and P. Fan [10] established that for $a=1, b=2$, and $0<s<\sqrt{5} / 5, W_{\alpha}+s W_{\alpha}^{2}$ is not hyponormal. On the other hand, it was shown in $[8$, Theorem 2] that a hyponormal weighted shift with three equal weights cannot be quadratically hyponormal without being flat: If $W_{\alpha}$ is quadratically hyponormal and $\alpha_{n}=\alpha_{n+1}=\alpha_{n+2}$ for some $n \geq 0$, then $\alpha_{1}=\alpha_{2}=\alpha_{3}=\cdots$, i.e., $W_{\alpha}$ is subnormal. Furthermore, in [8, Proposition 11] it was shown that, in the presence of quadratic hyponormality, two consecutive pairs of equal weights again force flatness, thereby subnormality. Y. Choi [5] improved this result, that is, if $W_{\alpha}$ is quadratically hyponormal and $\alpha_{n}=\alpha_{n+1}$ for some $n \geq 1$, then $W_{\alpha}$ is flat. Moreover, Y. Choi [5] also showed that if $W_{\alpha}$ is polynomially hyponormal and $\alpha_{n}=\alpha_{n+1}$ for some $n \geq 0$, then $W_{\alpha}$ is flat.

Proposition 5. (Propagation) Let $W_{\alpha}$ be a weighted shift with weight sequence $\left\{\alpha_{n}\right\}_{n=0}^{\infty}$.

(i) ([16, Theorem 6]) Let $W_{\alpha}$ be subnormal. If $\alpha_{n}=\alpha_{n+1}$ for some $n \geq 0$, then $\alpha$ is flat, i.e., $\alpha_{1}=\alpha_{2}=\alpha_{3}=\cdots$.

(ii) ([8, Corollary 6]) Let $W_{\alpha}$ be 2-hyponormal. If $\alpha_{n}=\alpha_{n+1}$ for some $n \geq 0$, then $\alpha$ is flat.

(iii) ([5, Theorem 1]) Let $W_{\alpha}$ be quadratically hyponormal. If $\alpha_{n}=$ $\alpha_{n+1}$ for some $n \geq 1$, then $\alpha$ is flat.

(iv) ([5, Theorem 2]) Let $W_{\alpha}$ be polynomially hyponormal. If $\alpha_{n}=$ $\alpha_{n+1}$ for some $n \geq 0$, then $\alpha$ is flat.

We now show outer propagation phenomena occurs for a norm attaining hyponormal weighted shift $W_{\alpha}$.

Corollary 6 . Let $W_{\alpha}$ be a norm attaining hyponormal weighted shift. Then $\alpha_{n}=\alpha_{n+1}=\cdots$ for some $n \geq 0$.

Proof. By Theorem 4, we have that $\left\|W_{\alpha}\right\|=\alpha_{n}=\max _{i} \alpha_{i}$ for some $n \geq 0$. But since $W_{\alpha}$ is hyponormal, the corresponding weight sequence is monotonically increasing. Thus, $\alpha_{n}=\alpha_{n+1}=\cdots$.

Moreover, we have:

CoRollary 7. (1) If $W_{\alpha}$ is a norm attaining quadratically hyponormal weighted shift. Then $\alpha$ is flat and hence $W_{\alpha}$ is subnormal.

(2) If $W_{\alpha}$ is a norm attaining 2-hyponormal weighted shift. Then $\alpha$ is flat and hence $W_{\alpha}$ is subnormal. 
Proof. It follows from Corollary 6 and Proposition 5.

Although the norm attaining operators are dense in $\mathcal{B}(\mathcal{H})$, we cannot expect that every hyponormal(or paranormal) operator is a norm attaining operator.

EXAMPLE 8. Let $\alpha \equiv\left\{\alpha_{n}\right\}_{n=0}^{\infty}$ be a strictly increasing bounded sequence. Then $W_{\alpha}$ is hyponormal (and hence paranormal), but not norm attaining.

Proof. Since the weight sequence is strictly increasing, $W_{\alpha}$ is clearly hyponormal. However, $\left\|W_{\alpha}\right\|^{2}$ cannot be in the point spectrum of $W_{\alpha}^{*} W_{\alpha}$ $=\operatorname{diag}\left\{\alpha_{0}^{2}, \alpha_{1}^{2}, \cdots\right\}$. Thus, by Lemma $1, W_{\alpha}$ is not norm attaining.

\section{References}

[1] A. Athavale, On joint hyponormality of operators, Proc. Amer. Math. Soc. 103 (1988), 417-423.

[2] E. Bishop, and R.R. Phelps, A proof that every Banach space is subreflexive, Bull. Amer. Math. Soc. 67 (1961), 97-98.

[3] J. Bram, Subnormal operators, Duke Math. J. 22(1955), 75-94.

[4] J. Bourgain, On dentability and the Bishop-Phelps property, Israel J. Math. 28 (1977), 265-271.

[5] Y. B. Choi, A propagation of quadratically hyponormal weighted shifts, Bull. Korean Math. Soc. 37 (2000), no. 2, 347-352.

[6] J. Conway, The Theory of Subnormal Operators, Math. Surveys Monogr. 36, Amer. Math. Soc., Providence, 1991.

[7] J.B. Conway and W. Szymanski, Linear combinations of hyponormal operators, Rocky Mountain J. Math. 18 (1988), 695-705.

[8] R.E. Curto, Quadratically hyponormal weighted shifts, Integral Equations Operator Theory, 13 (1990), 49-66.

[9] R. Curto, P. Muhly and J. Xia, Hyponormal pairs of commuting operators, Oper. Theory Adv. Appl. 35 (1988), 1-22.

[10] P. Fan, A note on hyponormal weighted shifts, Proc. Amer. Math. Soc. 92 (1984), 271-272.

[11] P.R. Halmos, A Hilbert space problem book, Springer, New York, 1982.

[12] A. Iwanik, Norm attaining operators on Lebesgue spaces, Pacific J. Math. 83 (1979), 381-386.

[13] J. Johnson, and J. Wolfe, Norm attaining operators, Studia Math. 65 (1979), $7-19$.

[14] A.D. Joshi, An example of a monotone shift which is not quadratically hyponormal, Math. Student 51 (1983), 193-194.

[15] J. Lindenstrauss, On operators which attain their norm, Israel J. Math. 1 (1963), 139-148. 
[16] J. Stamplli, Which weighted shifts are subnormal?, Pacific J. Math. 17 (1966), 367-379.

Department of Mathematics Education

Sangmyung University

Seoul 110-743, Republic of Korea

E-mail: jilee@smu.ac.kr 